\title{
How to Get More Transparency in Animal Experiments - Participation in CIRS-LAS
}

\author{
Enkelmann A and Bischoff $S^{*}$ \\ University Hospital Jena, Animal Welfare Unit, Germany \\ *Corresponding author: Bischoff S, University Hospital Jena, Animal Welfare Unit, Germany. \\ To Cite This Article: Enkelmann, A, Bischoff S, How to Get More Transparency in Animal Experiments - Participation in CIRS-LAS. 2020 - 10(5). \\ AJBSR.MS.ID.001546. DOI: 10.34297/AJBSR.2020.10.001546.
}

Received: 眥 October 02, 2020; Published: 战 October 21, 2020

\section{Opinion}

Considering the current state of science, the use of animals in experimental purposes cannot completely be replaced by i.e. chip technology or mathematical approaches. In Europe, every year about 10 million laboratory animals are still used in experiments, for teaching, or animal breeding for scientific purposes. Transparency and reproducibility are important tasks we must focus on laboratory animal science. In our everyday work we are faced with critical incidents, near accidents, or comparable negative events. The only way to deal with it is to recognize it, to analyze it, to discuss about it, and to find possible approaches to avoid repeating unsuccessful animal experiments. The task to use $3 \mathrm{R}$ guidelines or other animal welfare related resources is more and more asked to fulfill the responsible work with laboratory animals according the 3R principles [1,2].

Regarding the high number of publications based on animal experiments, we suppose also a certain amount of negative experiments. Literature research revealed that negative experiences gained from these experiments often get lost or are not referred in publications $[3,4]$. To make these negative experiences, critical incidents, or near accidents more visible, we developed a critical incident reporting system in laboratory animal science-CIRS-LAS [5]. This web-based platform is available for every person working with laboratory animals. On www.CIRS-LAS.de every person can share anonymously critical incidents and negative experiences from animal experiments or laboratory animal husbandry. The reporting is possible without registration and thus an easy access to the reporting system is possible. An expert panel as well as registered persons can comment the report and give assistance to improve processes or avoid further incidents in animal experiments.
The exchange of negative experiences between scientists, animal caretakers, technical assistants, and experts is a great step towards a constructive open dialogue about failures in laboratory animal science. This dialogue results in an enhanced transparency which is of great interest, not only in the scientific field, but also for the publicity. CIRS-LAS provides the platform for the exchange, the support, and the improvement of transparency in laboratory animal science.

CIRS-LAS is the world's first published web-based CIRS in laboratory animal science and plays an exemplary pioneering role in the implementation of the $3 \mathrm{R}$ principles reduce and refine: reduce the number of laboratory animals and improve animal safety. By a research in the CIRS-LAS database every person planning animal experiments can find out before, if the planned experimental setup has been associated with critical incidents or if recommendations or refinement methods in animal husbandry and animal welfare were discussed already. So, we would like to call you up to participate in CIRS-LAS. Only with your continuous support by reporting critical incidents or near accidents in animal experiments the animal welfare, the transparency, and thus the trust in animal experiments can enhance.

If you hesitate to participate due to worries about anonymity, we can assure you, that every report will be analyzed to ensure anonymity. Of course, CIRS-LAS works according to the actual data protection regulations. Further, your participation in CIRSLAS helps you to increase responsible handling of animals and improve quality and efficiency of your experiments. Therefore, the objective of CIRS-LAS is the detection of critical incidents in the entire range of laboratory animal science to help to avoid them in 
the future and therefore to reduce the repetition of unsuccessful animal experiments. Start your support for animal welfare right now-participate on www.CIRS-LAS.de

\section{Acknowledgement}

None.

\section{Conflict of Interest}

There is no conflict of interest.

\section{References}

1. Lewis DI (2019) Animal experimentation: implementation and application of the 3Rs. Emerg Top Life Sci 3(6): 675-679.

2. Russell WMS, Burch RL (1959) The Principles of Humane Experimental Technique. Methuen pp. 238.
3. Gerben ter Riet, Daniel A Korevaar, Marlies Leenaars, Peter J Sterk, Cornelis J F Van Noorden, et al. (2012) Publication bias in laboratory animal research: a survey on magnitude, drivers, consequences and potential solutions. PLoS One 7(9): e43404.

4. Wieschowski S, Svenja Biernot, Susanne Deutsch, Silke Glage, André Bleich, et al. (2019) Publication rates in animal research. Extent and characteristics of published and non-published animal studies followed up at two German university medical centres. Plos One 14(11): e0223758.

5. Bischoff S, David Trietschel, Astrid Enkelmann, René Schiffner, Pauline Estrade, et al. (2018) Learning from Negative Results-Critical Incident Reporting System in Laboratory Animal Science (CIRS-LAS.de). Anim Res Vet Sci 2(009). 